\title{
Maternal and fetal tuberous sclerosis complex: a case report questioning clinical approach
}

\author{
Özge Sürmeli Onay ${ }^{1 \oplus}$, Adviye Çakıl Sağlık ${ }^{1 \oplus}$, Pelin Köşger ${ }^{20}$, \\ Zeynep Nurhan Saraçoğlu ${ }^{3 \oplus}$, Uğur Toprak ${ }^{4 \oplus}$, Birsen Uçar ${ }^{2 \oplus}$, Ayşe Neslihan Tekin ${ }^{1 \oplus}$ \\ Divisions of ${ }^{1}$ Neonatology and ${ }^{2}$ Pediatric Cardiology, Department of Pediatrics, Departments of ${ }^{3}$ Dermatology and ${ }^{4}$ Radiology, Eskisehir \\ Osmangazi University, Faculty of Medicine, Eskisehir, Turkey.
}

\begin{abstract}
Background. Tuberous sclerosis complex (TSC) is an autosomal dominant neurocutaneous disease with multisystem involvement.

Case. Here, a mother and infant couple was presented with maternal and fetal TSC including demonstrative clinical findings and genetic analysis. The interesting point of this case report is that maternal and fetal TSC was identified after the mother gave birth to a child with a cardiac rhabdomyoma. The genetic analysis revealed a novel mutation which was the same in both the mother and her infant.

Conclusion. We would like to bring to the attention of clinicians this entity and to emphasize that maternal and fetal TSC can adversely affect maternal and fetal health, and deserves close follow up. Our recommendation is that if cardiac rhabdomyoma/cortical tuber/renal angiomyolipoma are present in prenatal ultrasonography, the parents should be evaluated for TSC.
\end{abstract}

Key words: maternal, fetal, tuberous sclerosis complex, rhabdomyoma, newborn.

Tuberous sclerosis complex (TSC, OMIM \#191100, \#613254) is an autosomal dominant disease characterized by benign tumor growth in many organs including the brain, heart, skin, eyes, kidneys, and lungs which presents with seizures, developmental delay, behavioral problems, skin abnormalities, and kidney disease. ${ }^{1}$ According to global statistics 1/6,000-10,000 people are born with TSC. ${ }^{2}$ Since molecular testing has become widely performed, the genetic examination of TSC was included in the Tuberous Sclerosis Complex Diagnostic Criteria Update: Recommendations of the 2012 International Tuberous Sclerosis Complex Consensus Conference. ${ }^{3}$ To date, many

$\triangle$ Özge Sürmeli Onay

ozgeonay79@gmail.com

Received 7th March 2019, revised 6th May 2019, accepted 21st June 2019.

This case report was presented as poster presentation in UNEKO 27, 3-7 April 2019, Antalya, Turkey. case reports about TSC have been published but reports dealing with maternal and fetal TSC are limited.-7 The interesting point of this case report is that maternal and fetal TSC was identified after the mother gave birth to a child with cardiac rhabdomyoma. Also, the genetic analysis revealed a novel mutation which was the same in both the mother and her infant. We would like to bring to the attention of clinicians this entity and to emphasize that maternal and fetal TSC can adversely affect maternal and fetal health.

\section{Case Report}

A female infant was born to a 28-year-old mother by emergency cesarean section due to severe preeclampsia at $25^{6}$ weeks of gestation with a birth weight of $860 \mathrm{~g}$ (50 percentile). The mother suffered from chronic hypertension and polycystic kidney disease. Her first pregnancy ended at $25^{4}$ weeks of gestation because of severe preeclampsia and fetal 
demise. There was no consanguinity between the parents. Apgar scores were $4 / 7 / 8$ at $1^{\text {th }}$, $5^{\text {th }}$ and $10^{\text {th }}$ minutes, respectively. The initial physical examination revealed respiratory distress. Surfactant replacement therapy was required and antibiotics (ampicillin, amicasin) were initiated. There was no cardiac murmur, hypotension or no necessity of inotropic support at follow up. At postnatal 48 hours, routinely performed transthoracic echocardiogram revealed multiple nonobstructive homogenous echogenic masses in interventricular septum and the papillary muscles of both ventricles protruding to the ventricular cavities and in the posterior wall of the left ventricle invading toward posterior atrioventricular groove consistent with rhabdomyomas (Fig. 1). Isolated premature supraventricular beats up to 2000 per day were observed during clinical followup. Cardiac rhabdomyomas in our patient were nonobstructive, hemodynamically not significant and did not cause any drug-resistant arrhythmia, so no medical or surgical treatment was initiated. We followed her on cardiac monitorization and spontaneous regresssion was detected on recurrent echocardiographic examinations. The diameters of the rhabdomyomas decreased 50 percent in eight months. The dermatological examination with Wood lamp, transfontanelle and abdominal ultrasound revealed no abnormalities associated with TSC. The infant remained hemodynamically stable throughout the hospitalisation, she was extubated at postnatal $9^{\text {th }}$ day, required no $\mathrm{O}_{2}$ support at postnatal $71^{\text {th }}$ day and discharged at postnatal $85^{\text {th }}$ day.

To evaluate maternal and fetal TSC, the mother of the infant was examined extensively. Maternal physical examination revealed facial angiofibromas on bilateral malar eminences and nasolabial folds, ungual fibromas, shagreen patch and hypomelanotic macule (Fig. 2) (Written informed consent was obtained from the mother). Cortical tubers and subependymal nodules were clearly identified on the cranial magnetic resonance images (MRI) (Fig. 3).

Although clinical diagnostic criteria for TSC was met in the mother, genetic testing was performed and the diagnosis was confirmed. The results of TSC 1 gene of the mother and infant revealed a heterozygous deletion mutation in TSC1 gene: NM-000368.4, c.2252-2253delAG (p.Lys751Serfs*2). To our knowledge, this mutation associated with TSC has not been reported previously.

\section{Discussion}

Tuberous sclerosis complex is an autosomal dominant neurocutaneous disease with
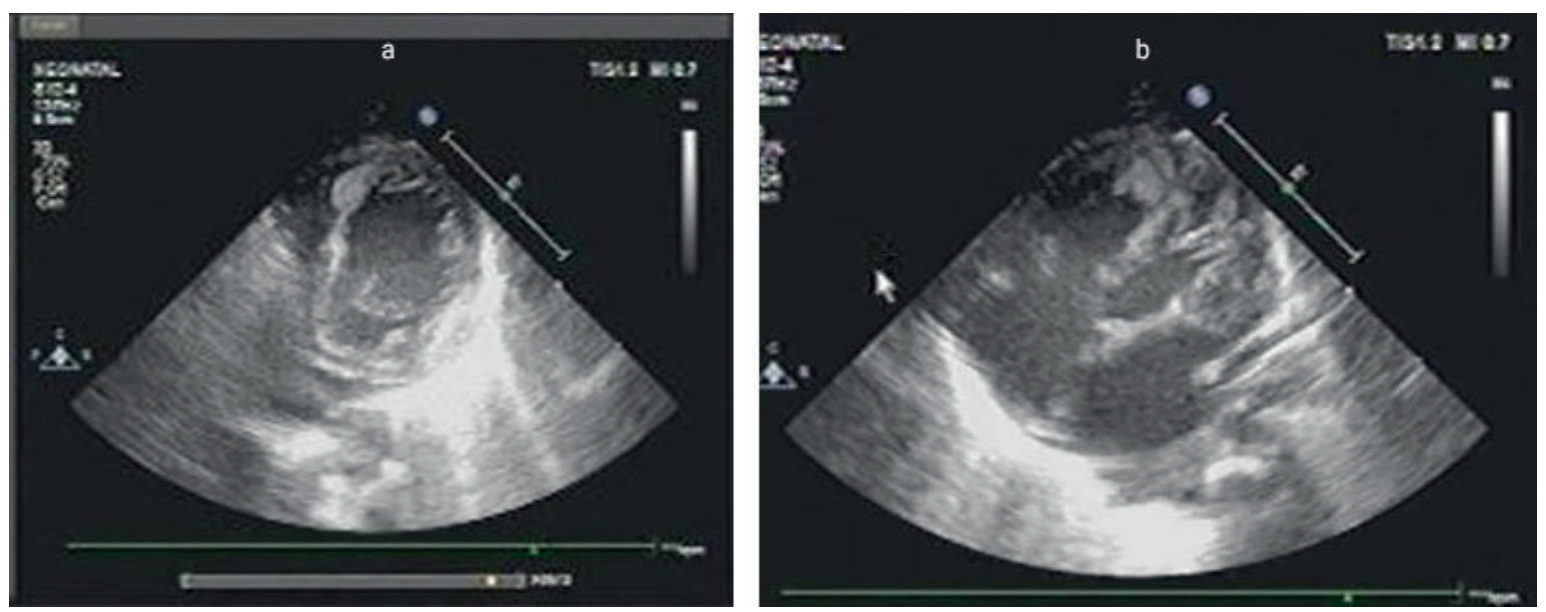

Fig. 1. Transthoracic echocardiography: (A) parasternal short axis view showing the mass inserted on the right side of the interventricular septum; and (B) apical four-chamber view of the mass inserted on the moderator band. 

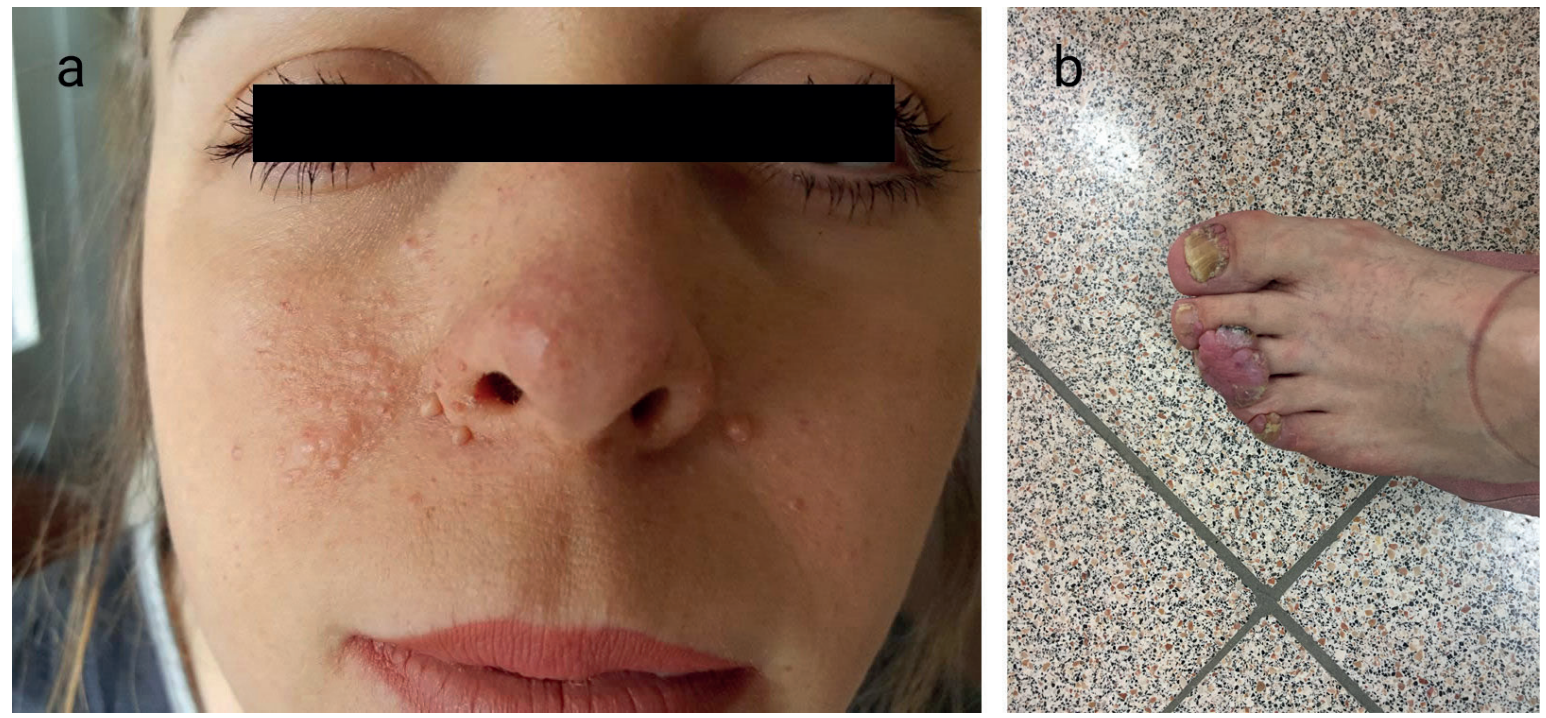

Fig. 2. (A).Facial angiofibromas on bilateral malar eminences and nasolabial folds (B). Subungual fibromas in the left foot.

multisystem involvement. Two tumor suppressor genes associated with TSC were demonstrated as follows; TSC1 is situated in region q34 of chromosome 9 and encodes the protein Hamartin, and TSC2 is situated in region p13 of chromosome 16 and encodes the protein Tuberin. $^{8}$ In the absence of clinical manifestations, the identification of a pathogenic mutation of TSC1 or TSC2 is adequate for the diagnosis of TSC. ${ }^{3}$ Tuberous sclerosis can come to exist by genetic transition from one parent with TSC or can result from a de novo genetic mutation. The possibility of inheritance for a child, if one of the parents has TSC is 50\%. ${ }^{5}$ Among TSC patients, pathogenic TSC2 gene mutations were reported in twothirds of all while pathogenic variants in TSC1 were determined in the remaining one-third. ${ }^{8} \mathrm{In}$ addition, there are TSC patients who have no mutation identified or have a variant mutation with unknown significance. Similarly, our cases have a new heterozygous deletion mutation which has not been reported to date. There has been no genotype-phenotype correlations including specific pathogenic variants of TSC1 reported yet. However, the presentation of TSC can be different among the family members with the same mutation because of the wide intrafamilial clinical variability. ${ }^{9}$
Cardiac rhabdomyoma is the most common cardiac tumor in infancy and childhood. Approximately $40 \%$ of patients with cardiac rhabdomyomas are diagnosed with TSC. Cardiac rhabdomyomas are present in $50-70 \%$ of patients with TSC. ${ }^{4}$ Renal manifestations of TSC commonly includes renal angiomyolipoma (AML) and renal cysts, rarely polycystic kidney disease or renal cell carcinoma. ${ }^{10}$ The prevalence of renal AML among TSC patients is 34 to $80 \%$. The clinical presentation of renal AML can be as variable as palpable mass, flank pain, urinary tract infections, spontaneous hemorrhage, arterial hypertension or kidney failure. ${ }^{11}$ However, the increase in circulating blood volume and renal blood flow during pregnancy, makes renal AML grow faster and leads to life threatening complications like rupture and retroperitoneal hemorrhage. ${ }^{12}$

This entity can adversely affect fetal and maternal morbidity and mortality. The complications of pregnant patients with TSC are preeclampsia, intrauterine growth retardation, preterm labor, preterm premature rupture of membrane, oligohydramnios, polyhydramnios, hydrops, abruption, hemorrhage from rupture of renal tumor, renal failure and fetal demise. ${ }^{6,7}$ In the literature, there are limited reports and 


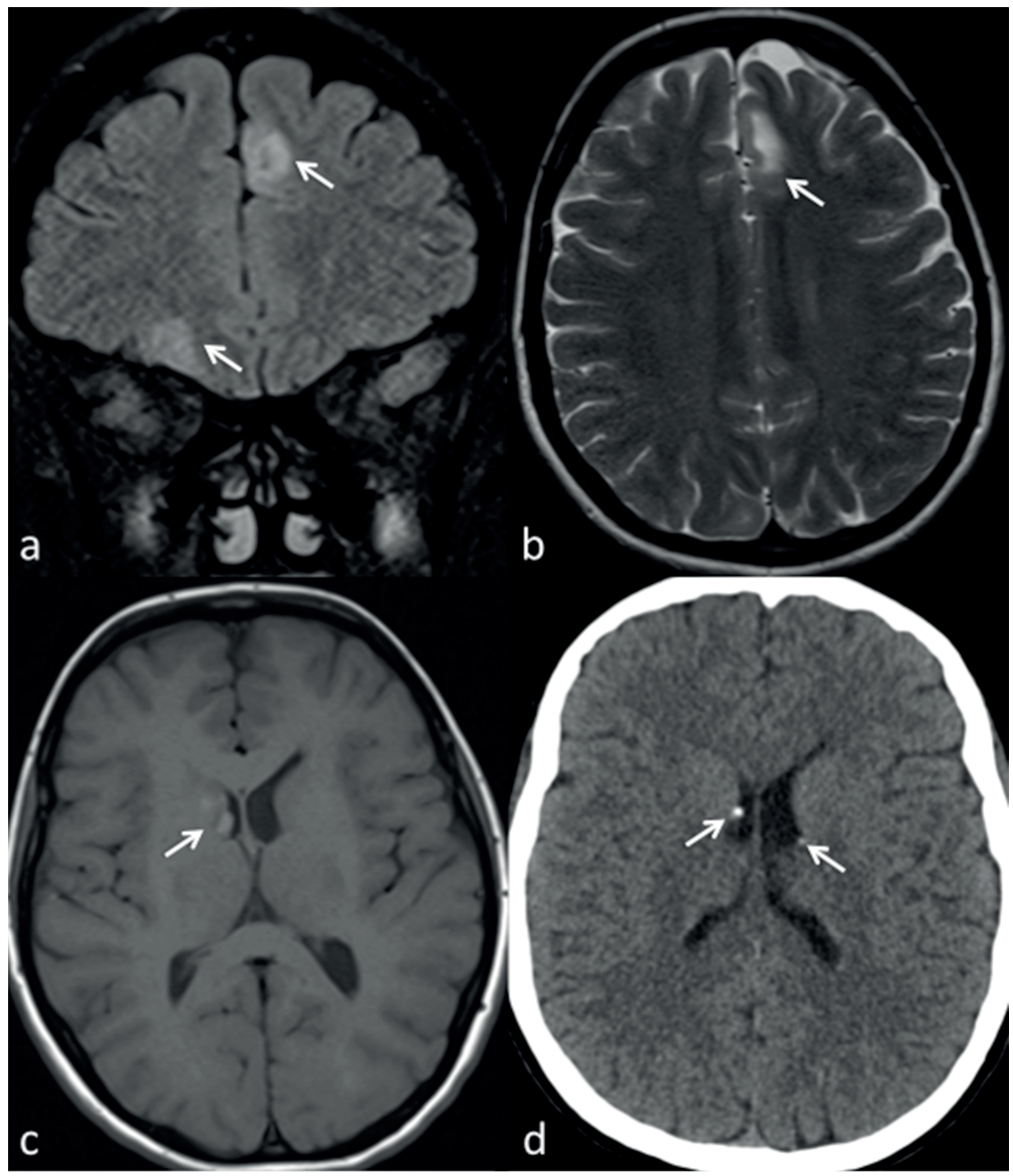

Fig. 3. (a) T2-weighted coronal FLAIR and (b) T2W axial MRI sequences demonstrate hyperintense cortical tubers (arrows) (c) T1W axial MRI image shows the subcortical, hyperintense subependymal nodule adjacent to the foramen Monro (arrow) (d) Axial computed tomography section shows subependymal calcified nodules (arrows).

inadequate awareness on maternal and fetal TSC. King et al. ${ }^{7}$ reported a pregnant patient with TSC who experienced the complications as preeclampsia, preterm labor, and fetal demise. Cardiac rhabdomyoma, intracranial tubers and hydrops fetalis were detected in her fetus. Similarly, kidney disease and chronic hypertension were the components of TSC 
in the mother of our case. So her pregnancy was complicated with preeclampsia, preterm labor and previous fetal demise. In another case report, cardiac rhabdomyomas were determined in both mother and fetus. Also, the left radical nephrectomy of the mother after delivery revealed renal AML and her infant died due to cardiac failure after delivery. ${ }^{4}$ Beyond these reports, there is a report presenting no intrapartum or peripartum complications in a couple of mother and infant having maternal and fetal TSC. ${ }^{6}$ However, Sharma et al. ${ }^{5}$ focused their attention to maternal and fetal TSC by questioning if obstetricians know enough about maternal and fetal TSC. The authors presented a pregnancy which ended with fetal demise due to fetal cardiac rhabdomyoma and emphasized genetic counseling for couples having a family history of TSC. Our experience taught us that early diagnosis of the mother can prevent complications of TSC and give a chance for genetic counselling before bearing a child.

With the advances in perinatal medicine, cardiac rhabdomyoma, cerebral lesions and renal AML can be identified in fetal ultrasonography or MRI. In the presence of these findings in prenatal ultrasonography, obstetricians should be aware of maternal and fetal TSC and carry out investigations for TSC. Maternal and fetal TSC deserves close follow up; if maternal TSC is known, the fetus should be evaluated with fetal Doppler echocardiography and if possible an MRI including brain and renal parenchyma. ${ }^{5}$ Reversely, our recommendation is that if cardiac rhabdomyoma/cortical tuber/renal AML are present in prenatal ultrasonography, the parents should be evaluated for TSC. Genetic counseling is necessary for parents with a family history of TSC because today, prenatal/ preimplantation diagnosis is possible. We believe that increasing awareness of maternal and fetal TSC will decrease the morbidity and mortality and increase the quality of life for patients with TSC.

In conclusion, it is noteworthy that the physical examination findings of the mother were overlooked despite many medical examinations for hypertension and pregnancy up until the age of 28 years. The delayed diagnosis of the mother shows the importance of performing a complete physical examination whatever the patient's complaint.

\section{Acknowledgements}

We thank to Associate Professor Kürşat Bora Çarman for his suggestions.

\section{REFERENCES}

1. Crino PB, Nathanson KL, Henske EP. The tuberous sclerosis complex. N Engl J Med 2006; 355: 13451356.

2. Hallett L, Foster T, Liu Z, Blieden M, Valentim J. Burden of disease and unmet needs in tuberous sclerosis complex with neurological manifestations: systematic review. Curr Med Res Opin 2011; 27: 1571-1583.

3. Northrup H, Krueger DA; International Tuberous Sclerosis Complex Consensus Group. Tuberous sclerosis complex diagnostic criteria update: Recommendations of the 2012 International Tuberous Sclerosis Complex Consensus Conference. Pediatr Neurol 2013; 49: 243-254

4. Zungsontiporn N, Tantrachoti P, Puwanant S.Foetal and maternal cardiac rhabdomyomas associated with tuberous sclerosis. Eur Heart J 2016; 37: 2036.

5. Sharma N, Sharma S, Thiek JL, Ahanthem SS, Kalita A, Lynser D. Maternal and fetal tuberous sclerosis: do we know enough as an obstetrician? J Reprod Infertil 2017; 18: 257-260

6. Gupta N, Singh N, Sarangi S, Dalmia S, Mittal S. Fetal cardiac rhabdomyoma with maternal tuberous sclerosis complicating pregnancy. Arch Gynecol Obstet 2008; 278: 169-170.

7. King JA, Stamilio DM. Maternal and fetal tuberous sclerosis complicating pregnancy: a case report and overview of the literature. Am J Perinatol 2005; 22: 103-108.

8. Peron A, Au KS, Northrup H. Genetics, genomics, and genotype-phenotype correlations of TSC: insights for clinical practice. Am J Med Genet C Semin Med Genet 2018; 178: 281-290.

9. Yang G, Shi ZN, Meng Y, et al. Phenotypic and genotypic characterization of Chinese children diagnosed with tuberous sclerosis complex. Clin Genet 2017; 91: 764-768. 
10. Koo KC, Kim WT, Ham WS, Lee JS, Ju HJ, Choi YD. Trends of presentation and clinical outcome of treated renal angiomyolipoma. Yonsei Med J 2010; 51: 728-734.

11. Bissler JJ, Kingswood JC. Renal angiomyolipomata. Kidney Int 2004; 66: 924-934.
12. Zapardiel I, Delafuente-Valero J, Bajo-Arenas JM. Renal angiomyolipoma during pregnancy: review of the literature. Gynecol Obstet Invest 2011; 72: 217219. 